\title{
Peritumoral eosinophils predict recurrence in colorectal cancer
}

\author{
Lars Harbaum ${ }^{1}$, Marion J Pollheimer ${ }^{2}$, Peter Kornprat ${ }^{3}$, Richard A Lindtner ${ }^{4}$, \\ Carsten Bokemeyer ${ }^{1}$ and Cord Langner ${ }^{2}$ \\ ${ }^{1}$ Department of Internal Medicine II, University Medical Center Hamburg-Eppendorf, Hamburg, Germany; \\ ${ }^{2}$ Institute of Pathology, Medical University of Graz, Graz, Austria; ${ }^{3}$ Department of Surgery, Medical University \\ of Graz, Graz, Austria and ${ }^{4}$ Department of Surgery, Medical University of Innsbruck, Innsbruck, Austria
}

\begin{abstract}
In colorectal cancer, the presence and extent of eosinophil granulocyte infiltration may render important prognostic information. However, it remains unclear whether an increasing number of eosinophils might simply be linked to the overall inflammatory cell reaction or represent a self-contained, antitumoral mechanism that needs to be documented and promoted therapeutically. Peri- and intratumoral eosinophil counts were retrospectively assessed in $\mathbf{3 8 1}$ primary colorectal cancers from randomly selected patients. Tumors were diagnosed in American Joint Committee on Cancer (AJCC)/Union Internationale Contre le Cancer (UICC) stage I in $21 \%$, stage II in $32 \%$, stage III in $33 \%$, and stage IV in $14 \%$. Presence and extent of eosinophils was related to various histopathological parameters as well as patients' outcome. Overall, peri- and intratumoral eosinophils were observed in $\mathbf{8 6}$ and $\mathbf{7 5 \%}$ cancer specimens. The peritumoral eosinophil count correlated strongly with the intratumoral eosinophil count $(R=0.69 ; P<0.001)$ and with the intensity of the overall inflammatory cell reaction $(R=0.318 ; P<0.001)$. Both increasing peri- and intratumoral eosinophil counts were significantly associated with lower $\mathrm{T}$ and $\mathrm{N}$ classification, better tumor differentiation, absence of vascular invasion, as well as improved progression-free and cancer-specific survival. However, only peritumoral eosinophils, but not intratumoral, were an independent prognosticator of favorable progression-free (hazard ratio 0.75 ; $95 \%$ confidence interval $0.58-0.98 ; P=0.04$ ) and cancer-specific survival (hazard ratio $0.7 ; 95 \%$ confidence interval $0.52-0.93 ; P=0.01$ ) independent of the intensity of overall inflammatory cell reaction. This was also found for patients with AJCC/ UICC stage II disease, wherein the presence of peritumoral eosinophils was significantly associated with favorable outcome. In conclusion, the number of peritumoral eosinophils had a significant favorable impact on prognosis of colorectal cancer patients independent of the overall tumor-associated inflammatory response. Evaluation of peritumoral eosinophils represents a promising readily assessable tool and should therefore routinely be commented on in the pathology report.
\end{abstract}

Modern Pathology (2015) 28, 403-413; doi:10.1038/modpathol.2014.104; published online 12 September 2014

Colorectal cancer is a global burden and ranges among the most common cancers and cancer-related causes of death worldwide. ${ }^{1}$ Stage according to the American Joint Committee on Cancer (AJCC)/Union Internationale Contre le Cancer (UICC) tumor node metastasis (TNM) system remains the most important prognostic parameter and guidance for therapeutic decision making. ${ }^{2}$ However, patients with an identical stage may experience considerably different clinical courses of disease. Thus,

Correspondence: Dr C Langner, MD, Institute of Pathology, Medical University of Graz, Auenbruggerplatz 25, 8036 Graz, Austria.

E-mail: cord.langner@medunigraz.at

Received 6 April 2014; revised 5 June 2014; accepted 15 June 2014; published online 12 September 2014 additional stage-independent pathologic parameters are warranted to identify patients who may benefit from adjuvant treatment. Although adjuvant chemotherapy is the standard approach in AJCC/UICC stage III patients, it is considered for AJCC/UICC stage II patients only in high-risk constellations, such as pT4 disease. ${ }^{1,2}$

Tumor-related local inflammation has been proposed as a hallmark of cancer and is emerging as a novel prognostic parameter. ${ }^{3,4}$ It is evident that multiple different cell types of the adaptive and innate immune system interact with cancer cells and may also have a prognostic value in colorectal cancers. ${ }^{3,5}$ However, a reliable measure of the antitumor local inflammatory cell reaction is yet to be integrated into routine pathology. ${ }^{3,5}$ Histopathology-based assessment of the overall 
local inflammatory cell reaction has been developed as a simple and reproducible grading scheme for estimation of the inflammatory cell reaction, which is independent from classical cancer-cell characteristics, such as morphology, cell of origin, molecular pathways, mutation status, and gene expression-based stratification. ${ }^{5,6}$ A pronounced local (in situ) inflammatory cell reaction in and around the tumor has been associated with favorable prognosis in colorectal cancers independent of AJCC/UICC stage. ${ }^{5}$

Eosinophil infiltration in the tumoral area, which is also called tumor-associated tissue eosinophilia, is an easy-assessable parameter in routine pathology. Increased numbers of eosinophils have been associated with disease recurrence and survival in patients with colorectal cancer. ${ }^{7-10}$ Furthermore, the occurrence of eosinophil infiltration has been linked to the overall inflammatory cell reaction. ${ }^{11}$ This raises the question whether the infiltration of eosinophils is a simple 'bystander' of an inflammatory host's reaction or might represent a selfcontained antitumoral mechanism that needs to be documented in routine pathology and promoted therapeutically?

The present analysis aims to assess the role of intratumoral and peritumoral eosinophils in regard of their association with various histopathological parameters and prognosis in colorectal cancer patients. Furthermore, we compared the prognostic impact of eosinophils with that of traditional and emerging histopathological parameters, such as vascular invasion, tumor budding, and overall inflammatory cell reaction, focusing on AJCC/UICC stage II disease.

\section{Materials and methods}

\section{Patient' Selection and Follow-Up Assessment}

During the period from 1 January 1984 to 31 December 2005, a total of 7909 colorectal cancers from 7564 patients (4095 males, 3469 females; ratio 1.2:1) were identified in the local colorectal cancer database of the Institute of Pathology (Medical University of Graz, Austria). Of these, 400 (5\%) patients were randomly sampled from January 1992 through December 2000 with the aim of obtaining identical adjuvant treatment modalities (see below) as well as a minimum of 5 years' follow-up.

The following patients were excluded: (i) those who underwent endoscopic polypectomy for lowrisk $\mathrm{T} 1$ cancer due to missing data regarding nodal status; (ii) patients who underwent neoadjuvant chemotherapy due to presumptive treatment-related changes in $\mathrm{T}$ classification; (iii) patients with synchronous or metachronous secondary colorectal cancer; and (iv) patients with competitive invasive cancers originating from other sites if metastatic deposits were not assessed by histology.
Finally, 381 patients out of 400 (95\%) were included for review pathology (166 males, 215 females; ratio 1:1.5; mean age 68.5 years). Tumors were located on the right side in 107 (cecum to transverse colon), on the left side in 110 (flexura lienalis to sigmoid colon), and in the rectum in 164 cases.

Adjuvant chemotherapy was guided by AJCC/ UICC stage: stage I and II patients did not receive adjuvant therapy, whereas stage III patients were given 5-fluorouracil/folinic acid-based chemotherapy according to the Mayo Clinic protocol. ${ }^{12}$

Follow-up included laboratory testing (including blood count, liver enzymes, and tumor markers (carcinoembryonic antigen and carbohydrate antigen 19-9)) at 3-month intervals (extended to 6 months after 3 years) and chest X-ray and abdominal ultrasound at 6-month intervals (extended to 12 months after 3 years). Patients with rectal cancer underwent computerized tomography every 12 months.

Progressive disease was defined as either local recurrence (any detectable local disease, occurring either alone or in conjunction with generalized recurrence) or systemic recurrence (any detectable disease, except local recurrence).

Institutional review board approval was received from the local ethics committee.

\section{Histopathology}

$\mathrm{T}$ and $\mathrm{N}$ classification were assessed according to the AJCC/UICC 2009 issue of the TNM classification. ${ }^{13}$ Histological tumor type and differentiation (grade) were assessed according to the 2010 edition of the World Health Organization classification. ${ }^{14}$

Vascular invasion, including both venous and lymphatic invasion, was considered positive when tumor cells or tumor cell thrombi were observed within an endothelium-lined space. Special care was taken to differentiate endothelial cells from retraction artifacts lined by fibroblasts. When carcinoma cells were present in vessels with a thick vascular wall and red blood cells in the lumen, this was considered blood vessel invasion. Immunohistochemistry for endothelial cells was not routinely done, in keeping with standard practice.

The extent of tumor budding was assessed on hematoxylin and eosin-stained slides as described previously by Ueno et al: ${ }^{15}$ in a field in which budding intensity was maximal, the number of isolated single cells or small clusters of less than five cells scattered in the stroma at the invasive tumor margin was counted using a $\times 20$ objective lens in a field measuring $0.95 \mathrm{~mm}^{2}$ (Olympus BX45, Tokyo, Japan). The intensity of budding was recorded as follows: score 1 ( $<5$ budding foci), score 2 (refs 5-8,16), score 3 (refs 9-15,17-19), and score $4(\geq 20)$. Tumor budding was summarized as low (score 1 and 2) and high (score 3 and 4).

The number of eosinophils was assessed separately within the tumor center (intratumoral) and in 
the stroma at the invasive tumor margin (peritumoral) on hematoxylin and eosin-stained slides. Eosinophils were counted and graded as described previously by Fernandez-Acenero et $a l^{8}$ using a $\times 40$ objective lens in a high power field measuring $0.24 \mathrm{~mm}^{2}$ (Olympus BX45): absence of eosinophils, low eosinophil count $\left(<10 / 0.24 \mathrm{~mm}^{2}\right)$, intermediate eosinophil count (10-50/0.24 $\left.\mathrm{mm}^{2}\right)$, and high eosinophil count $\left(>50 / 0.24 \mathrm{~mm}^{2}\right)$. Figure 1 shows an example of a colorectal adenocarcinoma with high peritumoral eosinophil count.

The intensity of overall inflammatory cell reaction at the invasive margin was assessed as described by Klintrup et $a l^{6}$ on hematoxylin and eosin-stained slides: A score of 0 indicated there was no increase in inflammatory cells, a score of 1 represented a mild or patchy increase, a score of 2 a prominent inflammatory reaction, and a score of 3 a florid 'cuplike' inflammatory infiltrate.

Review pathology was performed independently by two gastrointestinal pathologists (MJP and CL), who were blinded regarding clinical data. Discrepancies were resolved by simultaneous re-examination of the slides by both investigators using a double-headed microscope.

\section{Statistical Analysis}

Associations between eosinophil count and tumor parameters were analyzed using $\chi^{2}$-test. Spearman's rank correlation was performed to assess correlation of nonparametric data. Progression-free and cancerspecific survival was assessed using the KaplanMeier method. Significance among patient groups was calculated by using the log-rank test. To determine hazard ratios, we used Cox proportional hazards regression model. All reported test were two sided and $P$-values were significant at levels $<0.05$. All statistical calculations were performed using SPSS statistics version 20 (IBM, Armonk, NY, USA).

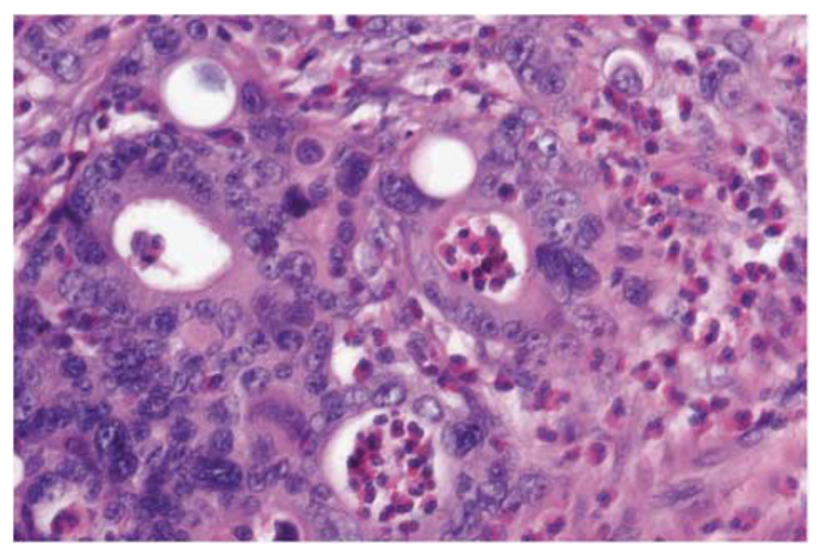

Figure 1 High eosinophil count at the invasive margin. A colorectal adenocarcinoma showing high peritumoral eosinophil count (10-50 eosinophils $/ 0.24 \mathrm{~mm}^{2}$ ) at the invasive tumor margin (hematoxylin and eosin stain; original $\times 250$ ).

\section{Results}

Increasing Peritumoral and Intratumoral Eosinophil Counts are Associated with Favorable Tumor Parameters

Peritumoral eosinophils were observed in 328 (86\%) and intratumoral eosinophils in 284 (75\%) colorectal cancer specimens. Herein, the peritumoral eosinophil count correlated strongly with the intratumoral eosinophil count $(R=0.69 ; P<0.001)$. Increasing peritumoral eosinophil counts were associated significantly with lower $\mathrm{T}$ and $\mathrm{N}$ classification, better tumor differentiation as well as the absence of lymphatic and venous invasion. Similar data were observed for increasing intratumoral eosinophil counts-although not for $\mathrm{N}$ classification. Table 1 summarizes the distribution of intratumoral and peritumoral eosinophil counts related to different tumor characteristics.

\section{Peritumoral and Intratumoral Eosinophils Correlate with Overall Inflammatory Cell Reaction}

An increased overall inflammatory cell reaction was observed in 370 (97\%) cancers (score 1-3). Herein, $156(42 \%)$ showed a mild or patchy increase (score 1), $146(39 \%)$ a prominent (score 2$)$, and $68(19 \%)$ a florid 'cup-like' inflammatory reaction (score 3). Increasing inflammatory reaction correlated positively with increasing peritumoral $(R=0.318$; $P<0.001)$ and intratumoral $(R=0.214 ; P<0.001)$ eosinophil count. However, the inflammatory cell reaction varied among tumors with identical peritumoral eosinophil counts. Thus, tumors lacking peritumoral eosinophils showed a prominent to florid overall inflammatory cell reaction (score 2 or 3) in $16(31 \%)$ cases (Figure 2a). Likewise, tumors with a high peritumoral eosinophil count demonstrated only a mild inflammatory cell reaction (score $<2)$ in $4(20 \%)$ cases (Figure $2 b)$.

\section{Increasing Peritumoral and Intratumoral Eosinophil Counts are Associated with Progression-Free and Cancer-Specific Survival}

Follow-up data were available for 350 out of 381 (92\%) patients. Median follow-up was 45 months (range 1-182). After a median of 15 months (range 0-88), progression (local or systemic recurrence) occurred in 141 (37\%) patients. Of these, 118 patients died from cancer, 11 patients were alive with metastatic disease, 7 patients showed no further progress after metastasectomy, and 5 patients presented initially in poor clinical condition due to advanced disease and died within 30 days after surgery.

Disease progression occurred in $29(62 \%)$ patients with tumors lacking peritumoral eosinophils, in 86 $(42 \%)$ with low peritumoral eosinophil count, in $25(32 \%)$ with intermediate eosinophil count, and in $1(5 \%)$ case with high eosinophil count $(P<0.001$; 
Table 1 Associations of eosinophil count with different tumor parameters

\begin{tabular}{|c|c|c|c|c|c|c|c|c|c|c|c|}
\hline \multirow[b]{2}{*}{ Tumor parameters } & & \multicolumn{5}{|c|}{ Peritumoral eosinophil count } & \multicolumn{5}{|c|}{ Intratumoral eosinophil count } \\
\hline & & $\begin{array}{c}\text { Absent } \\
\mathrm{n}=53\end{array}$ & $\begin{array}{c}\text { Low } \\
\mathrm{n}=227\end{array}$ & $\begin{array}{l}\text { Intermediate } \\
\mathrm{n}=81\end{array}$ & $\begin{array}{c}\text { High } \\
\mathrm{n}=20\end{array}$ & $\mathrm{P}$-value & $\begin{array}{c}\text { Absent } \\
\mathrm{n}=97\end{array}$ & $\begin{array}{c}\text { Low } \\
\mathrm{n}=237\end{array}$ & $\begin{array}{c}\text { Intermediate } \\
\mathrm{n}=44\end{array}$ & $\begin{array}{l}\text { High } \\
\mathrm{n}=3\end{array}$ & $\mathrm{P}$-value \\
\hline \multicolumn{12}{|l|}{ T classification } \\
\hline T1 & $n=28$ & $1(2 \%)$ & $16(7 \%)$ & $8(10 \%)$ & $3(15 \%)$ & $<0.01$ & $3(3 \%)$ & $19(8 \%)$ & $6(14 \%)$ & - & $<0.01$ \\
\hline $\mathrm{T} 2$ & $n=70$ & $5(9 \%)$ & $35(15 \%)$ & $22(27 \%)$ & $8(40 \%)$ & & $10(10 \%)$ & $46(19 \%)$ & $13(29 \%)$ & $1(33 \%)$ & \\
\hline T3 & $n=218$ & $29(55 \%)$ & $136(60 \%)$ & $44(54 \%)$ & $9(45 \%)$ & & $55(57 \%)$ & $137(58 \%)$ & $24(55 \%)$ & $2(67 \%)$ & \\
\hline $\mathrm{T} 4$ & $n=65$ & $18(34 \%)$ & $40(18 \%)$ & $7(9 \%)$ & - & & $29(30 \%)$ & $35(15 \%)$ & $1(2 \%)$ & - & \\
\hline \multicolumn{12}{|l|}{$N$ classification } \\
\hline No & $n=213$ & $20(38 \%)$ & $125(55 \%)$ & $52(64 \%)$ & $16(80 \%)$ & $<0.01$ & $45(46 \%)$ & $137(58 \%)$ & $29(66 \%)$ & $2(67 \%)$ & 0.16 \\
\hline N1 & $n=83$ & $12(23 \%)$ & $50(22 \%)$ & $17(21 \%)$ & $4(20 \%)$ & & $21(22 \%)$ & $53(22 \%)$ & $8(18 \%)$ & $1(33 \%)$ & \\
\hline N2 & $n=85$ & $21(40 \%)$ & $52(23 \%)$ & $12(15 \%)$ & - & & $31(32 \%)$ & $47(20 \%)$ & $7(16 \%)$ & - & \\
\hline \multicolumn{12}{|l|}{ AJCC/UICC stage } \\
\hline I & $n=81$ & $2(4 \%)$ & $44(19 \%)$ & $25(31 \%)$ & $10(50 \%)$ & $<0.01$ & $7(7 \%)$ & $57(24 \%)$ & $16(36 \%)$ & $1(33 \%)$ & $<0.01$ \\
\hline II & $n=120$ & $15(28 \%)$ & $74(33 \%)$ & $25(31 \%)$ & $6(30 \%)$ & & $32(33 \%)$ & $74(31 \%)$ & $13(30 \%)$ & $1(33 \%)$ & \\
\hline III & $n=126$ & $21(40 \%)$ & $77(34 \%)$ & $24(30 \%)$ & $4(20 \%)$ & & $36(37 \%)$ & $77(33 \%)$ & $12(27 \%)$ & $1(33 \%)$ & \\
\hline IV & $n=54$ & $15(28 \%)$ & $32(14 \%)$ & $7(9 \%)$ & - & & $22(23 \%)$ & $29(12 \%)$ & $3(7 \%)$ & - & \\
\hline \multicolumn{12}{|c|}{ Tumor differentiation } \\
\hline G1 & $n=121$ & $6(11 \%)$ & $73(32 \%)$ & $30(37 \%)$ & $12(60 \%)$ & $<0.01$ & $15(16 \%)$ & $87(37 \%)$ & $17(39 \%)$ & $2(67 \%)$ & $<0.01$ \\
\hline G2 & $n=138$ & $23(43 \%)$ & $86(38 \%)$ & 27 (33\%) & $2(10 \%)$ & & $42(43 \%)$ & $83(35 \%)$ & $13(30 \%)$ & - & \\
\hline G3 & $n=122$ & $24(45 \%)$ & $68(30 \%)$ & $24(30 \%)$ & $6(30 \%)$ & & $40(41 \%)$ & $67(28 \%)$ & $14(32 \%)$ & $1(33 \%)$ & \\
\hline \multicolumn{12}{|l|}{ Histology } \\
\hline Adenocarcinoma & $n=317$ & $38(72 \%)$ & $191(84 \%)$ & $70(86 \%)$ & $18(90 \%)$ & 0.09 & $73(75 \%)$ & $204(86 \%)$ & $38(86 \%)$ & $2(66 \%)$ & 0.08 \\
\hline Others $^{\mathrm{a}}$ & $n=64$ & $15(28 \%)$ & $36(16 \%)$ & $11(14 \%)$ & $2(10 \%)$ & & $24(25 \%)$ & $33(14 \%)$ & $6(14 \%)$ & $1(33 \%)$ & \\
\hline \multicolumn{12}{|l|}{ Lvmphatic invasion } \\
\hline Negative & $n=255$ & $27(51 \%)$ & $152(67 \%)$ & $59(73 \%)$ & $17(85 \%)$ & 0.02 & $49(51 \%)$ & $171(72 \%)$ & $32(73 \%)$ & $3(100 \%)$ & $<0.01$ \\
\hline Positive & $n=126$ & $26(49 \%)$ & $75(33 \%)$ & $22(27 \%)$ & $3(15 \%)$ & & $48(49 \%)$ & $66(28 \%)$ & $12(27 \%)$ & - & \\
\hline \multicolumn{12}{|l|}{ Venous invasion } \\
\hline Negative & $n=294$ & $31(59 \%)$ & $181(80 \%)$ & $65(80 \%)$ & $17(80 \%)$ & $<0.01$ & $61(63 \%)$ & $195(82 \%)$ & $35(80 \%)$ & $3(100 \%)$ & $<0.01$ \\
\hline Positive & $n=87$ & $2242 \%$ ) & $46(20 \%)$ & $16(20 \%)$ & $3(15 \%)$ & & $36(37 \%)$ & $42(18 \%)$ & $9(21 \%)$ & - & \\
\hline \multicolumn{12}{|l|}{ Tumor budding } \\
\hline Low & $n=221$ & $18(34 \%)$ & $132(58 \%)$ & $53(65 \%)$ & $18(90 \%)$ & $<0.01$ & $37(38 \%)$ & $150(63 \%)$ & $31(70 \%)$ & $3(100 \%)$ & $<0.01$ \\
\hline High & $n=160$ & $35(66 \%)$ & $95(42 \%)$ & $28(35 \%)$ & $2(10 \%)$ & & $60(62 \%)$ & $87(37 \%)$ & $13(30 \%)$ & - & \\
\hline
\end{tabular}

Abbreviations: AJCC, American Joint Committee on Cancer; UICC, Union Internationale Contre le Cancer.

$P$-values are two sided and were calculated by $\chi^{2}$-test.

${ }^{a}$ Including mucinous adenocarcinomas, undifferentiated, signet-ring cell and medullary carcinoma, and adenosquamous, mixed endocrineexocrine carcinoma.

Figure 3a). Actuarial 5-year progression-free survival rates were 40, 57, 67 and 95\%, respectively. Notably, progression occurred in only 1 out of 20 cases with high peritumoral eosinophil count, yet only 8 out of these 20 cases $(40 \%)$ showed a florid overall inflammatory cell reaction (Figure 2b). In addition, $25(53 \%)$ died from disease with tumors lacking peritumoral eosinophils, $70(34 \%)$ with low peritumoral eosinophil count, $21(27 \%)$ with intermediate eosinophil count, and $1(5 \%)$ patient with high eosinophil count $(P<0.001$; Figure 3b). Actuarial 5-year cancer-specific survival rates were 47, 65, 75 and $95 \%$, respectively. Similar results were observed for an increasing intratumoral eosinophil count (data not shown), wherein a higher number of eosinophils was associated significantly with progression-free as well as cancer-specific survival (both $P<0.001$; Figures 3c and d).

\section{Peritumoral Eosinophils are an Independent Prognostic Parameter}

In Cox proportional hazards regression analyses comparing increasing peritumoral and intratumoral eosinophil counts, only peritumoral eosinophils, but not intratumoral, were significantly associated with progression-free (hazard ratio 0.64; 95\% confidence interval $0.46-0.92 ; P=0.01$ ) as well as cancer-specific survival (hazard ratio 0.64; 95\% confidence interval $0.44-0.94 ; P=0.02$ ).

In a second step, peritumoral eosinophil count was compared with well-established prognostic tumor parameters. In Cox proportional hazards regression analyses including $\mathrm{T}$ and $\mathrm{N}$ classification, tumor differentiation, lymphatic and venous invasion, tumor budding as well as intensity of overall inflammatory cell reaction, an increasing peritumoral 

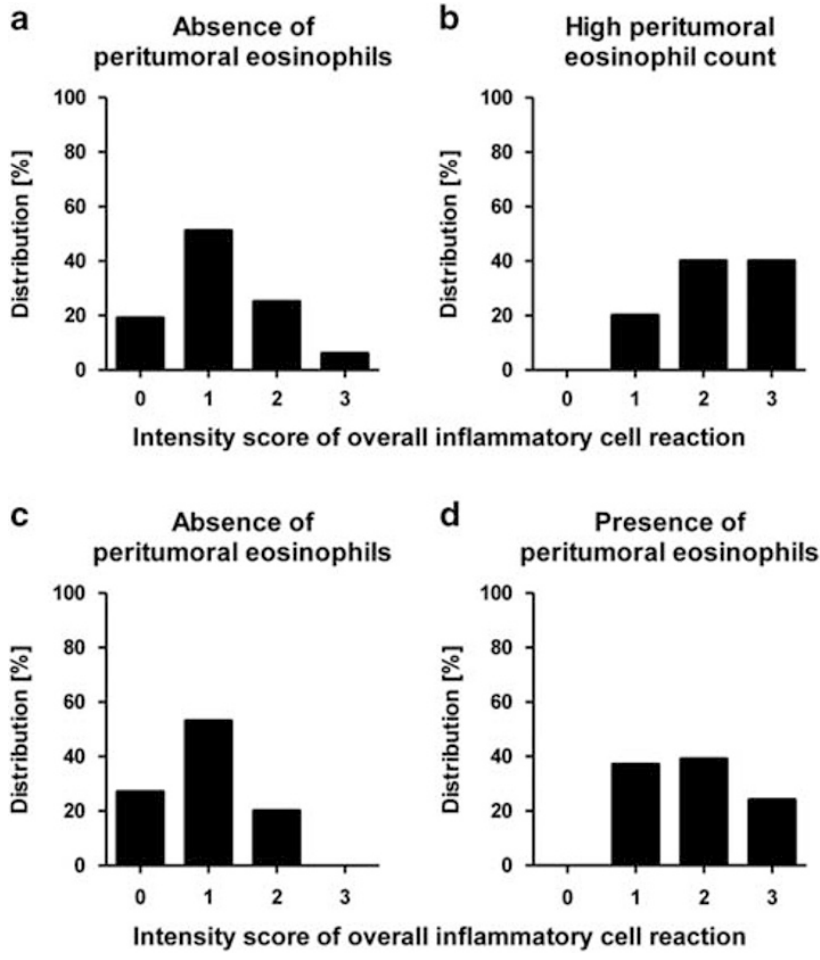

Figure 2 Distribution of the intensity of the overall inflammatory cell reaction among tumors with different eosinophil counts. Distribution of the overall inflammatory cell reaction in tumors lacking peritumoral eosinophils $(n=53$; (a)) and in tumors with high peritumoral eosinophil count $(n=20$; (b)). Distribution of overall inflammatory cell reaction considering only tumors diagnosed in AJCC/UICC stage II disease either lacking peritumoral eosinophils $(n=15$; (c)) or with presence of peritumoral eosinophils $(n=105$; (d)). AJCC, American Joint Committee on Cancer; UICC, Union Internationale Contre le Cancer.

eosinophil count was independently associated with favorable progression-free (hazard ratio 0.75 ; 95\% confidence interval $0.58-0.98 ; P=0.04)$ and cancer-specific survival (hazard ratio $0.7 ; 95 \%$ confidence interval $0.53-0.93 ; P=0.01$; Table 2). Importantly, an increasing peritumoral eosinophil count predicted survival independent of the intensity of overall inflammatory cell reaction, although the latter was associated with progression-free (hazard ratio $0.71 ; 95 \%$ confidence interval $0.53-$ $0.89 ; P=0.003$ ) and cancer-specific survival (hazard ratio $0.65 ; 95 \%$ confidence interval $0.50-0.83$; $P=0.001)$ in univariable analyses.

Presence of Peritumoral Eosinophils is an Independent Prognostic Parameter in Patients with AJCC/UICC Stage II Colorectal Cancer

AJCC/UICC stage II disease was diagnosed in 120 $(32 \%)$ cases. Progression occurred in $21(19 \%)$ cases and $17(16 \%)$ patients died of disease.

Survival analyses restricted to patients with AJCC/UICC stage II cancers revealed no significant association of the four-tiered peritumoral eosinophil count with progression-free or cancer-specific survival, although the survival curves dissociated similarly (data not shown). However, when tumors were stratified according to the absence or presence of peritumoral eosinophils, presence of peritumoral eosinophils was significantly associated with favorable progression-free and cancer-specific survival. Thus, progression occurred in $6(43 \%)$ cases with tumors lacking peritumoral eosinophils compared with $15(16 \%)$ cases with tumors showing peritumoral eosinophils $(P=0.016$; Figure 4a). Actuarial 5 -year progression-free survival rates were 63 and $87 \%$. In addition, $5(36 \%)$ patients with tumors lacking peritumoral, yet only $12(13 \%)$ cases with tumors showing peritumoral eosinophils died of disease $(P=0.035$; Figure $4 \mathrm{~b})$. Actuarial 5-year cancer-specific survival rates were 70 and $90 \%$. Although associated with unfavorable survival, more than $50 \%$ of tumors (11 out of 15) lacking peritumoral eosinophils showed an increased inflammatory cell reaction (score $>0$; Figure 2c). Furthermore, only $24 \%$ of tumors (25 out of 105), in which peritumoral eosinophils were found, showed a florid inflammatory cell reaction (score 3; Figure 2d).

In Cox proportional hazards regression analyses, presence of peritumoral eosinophils was associated with progression-free (hazard ratio 0.24 ; $95 \%$ confidence interval $0.07-0.87 ; P=0.03)$ and cancerspecific survival (hazard ratio 0.25 ; $95 \%$ confidence interval 0.06-1.02; $P=0.05$ ) independent of $\mathrm{T}$ and $\mathrm{N}$ classification, tumor differentiation, lymphatic and venous invasion, tumor budding as well as intensity of overall inflammatory cell reaction (Table 3).

Combined Analysis of Peritumoral Eosinophils and Tumor Budding is associated with Progression-Free and Cancer-Specific Survival-Overall and in AJCC/ UICC Stage II Colorectal Cancers

Although an increasing peritumoral eosinophil count represented a favorable tumor parameter, high extent of tumor cell budding was associated significantly with poor survival of patients (Tables 2 and 3). The peritumoral eosinophil count correlated inversely with the extent of tumor budding $(R=-0.185 ; P<0.001)$.

To evaluate the combined effect of these diametrical processes, we dichotomized both parameters and classified the tumors into three categories. Hereby, $71(19 \%)$ tumors showed intermediate-tohigh peritumoral eosinophil count and low extent of tumor budding. These patients had a favorable progression-free $(P<0.001$; Figure $5 \mathrm{a})$ and cancerspecific survival $(P<0.001$; Figure $5 \mathrm{~b})$. Progression occurred in only $9(13 \%)$ patients and $6(9 \%)$ patients died of the disease. The actuarial 5-year progression-free survival rate was $84 \%$ and cancerspecific survival rate $89 \%$. On the contrary, $130(34 \%)$ tumors demonstrated absent-to-low peritumoral 

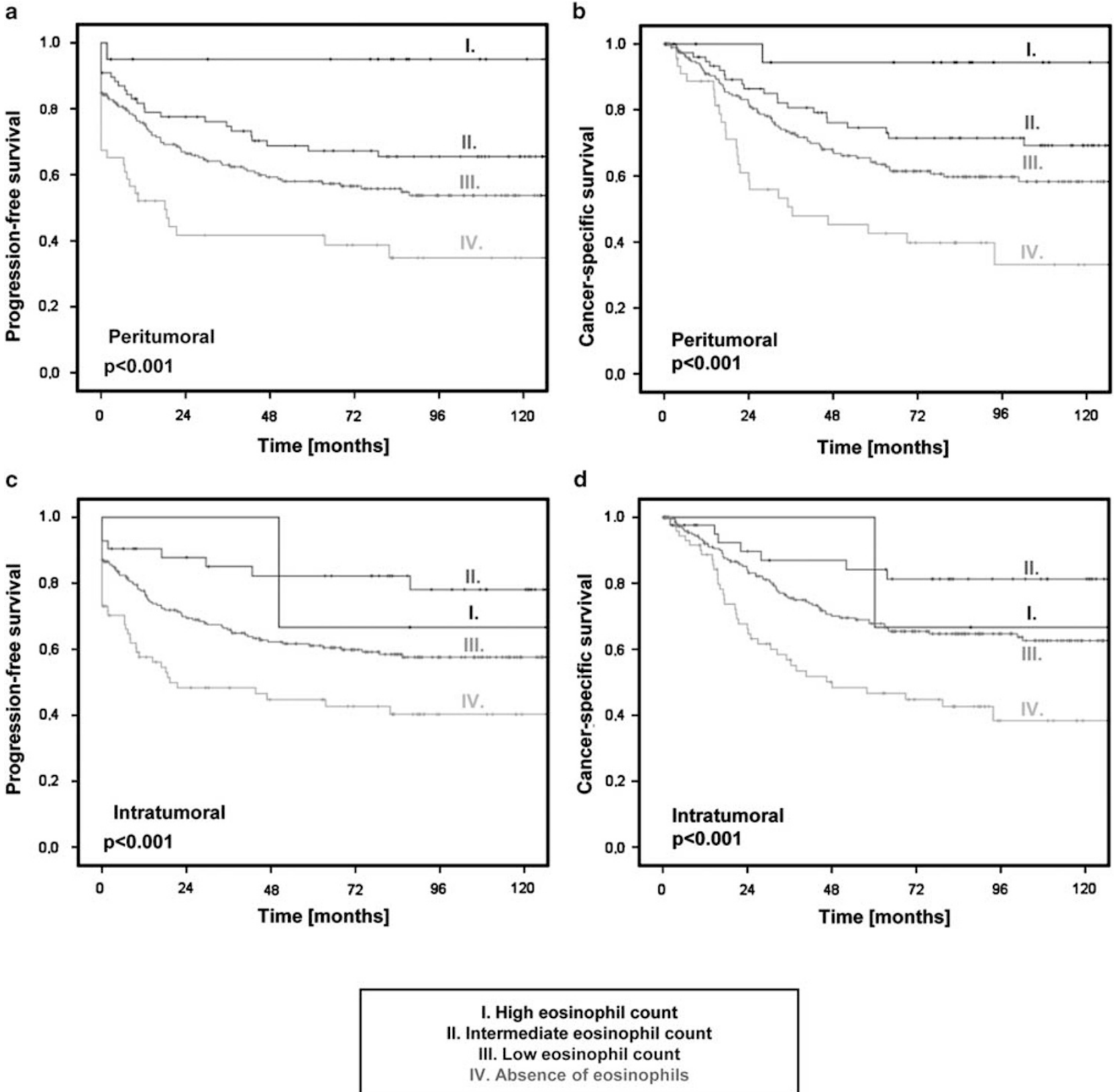

Figure 3 Peri- and intratumoral eosinophil count is associated with survival in colorectal cancer. Kaplan-Meier curves showing progression-free (a) and cancer-specific survival (b) according to the peritumoral eosinophil count, as well as progression-free (c) and cancer-specific survival (d) according to the intratumoral eosinophil count. Comparisons were calculated using log-rank test. $P$-values are two sided.

eosinophil count and high extent of tumor budding. These patients had a poor progression-free and cancer-specific survival. Progression occurred in $72(64 \%)$ patients and $63(55 \%)$ patients died of the disease. The actuarial 5-year progression-free survival rate was only $29 \%$ and cancer-specific survival rate $32 \%$.

A similar stratification of survival by this combined parameter was evident in patients with AJCC/ UICC stage II colorectal cancers. Patients with tumors showing an intermediate-to-high peritumoral eosinophil count and low extent of tumor budding demonstrated favorable and patients with tumors showing an absent-to-low peritumoral eosinophil count and high extent of tumor budding poor progression-free $(P=0.08$; Figure $5 \mathrm{c})$ and cancerspecific survival $(P=0.012$; Figure $5 \mathrm{~d})$. Actuarial 5-year progression-free survival rates were 82 and $66 \%$ and actuarial 5-year cancer-specific survival rates 87 and $60 \%$, respectively. 
Table 2 Cox proportional hazards regression model assessing the prognostic significance of increasing peritumoral eosinophil count in conjunction with other well-established tumor parameters regarding progression-free and cancer-specific survival in patients with colorectal cancers $(n=350)$

\begin{tabular}{|c|c|c|c|c|c|c|}
\hline & \multicolumn{3}{|c|}{ Progression-free survival } & \multicolumn{3}{|c|}{ Cancer-specific survival } \\
\hline & Hazard ratio & $95 \% C I$ & P-value & Hazard ratio & $95 \% C I$ & P-value \\
\hline Age $>70$ years & 0.98 & $0.70-1.40$ & 0.93 & 1.32 & $0.90-1.93$ & 0.15 \\
\hline Female gender & 1.12 & $0.79-1.59$ & 0.53 & 1.17 & $0.79-1.73$ & 0.42 \\
\hline Increasing $\mathrm{T}$ classification & 1.87 & $1.37-2.55$ & $<0.01$ & 1.80 & $1.28-2.53$ & $<0.01$ \\
\hline Presence of nodal disease ( $\mathrm{N}$ positive) & 2.49 & $1.60-3.88$ & $<0.01$ & 2.47 & $1.53-3.99$ & $<0.01$ \\
\hline Poor tumor differentiation (G3) & 1.03 & $0.71-1.49$ & 0.89 & 1.75 & $1.19-2.57$ & 0.01 \\
\hline Presence of lymphatic invasion & 1.04 & $0.71-1.53$ & 0.85 & 1.03 & $0.69-1.56$ & 0.88 \\
\hline Presence of venous invasion & 1.55 & $1.04-2.31$ & 0.03 & 1.99 & $1.31-3.04$ & $<0.01$ \\
\hline High tumor budding & 1.38 & $1.13-1.68$ & $<0.01$ & 1.42 & $1.16-1.77$ & $<0.01$ \\
\hline Increasing intensity of overall inflammatory cell reaction & 1.04 & $0.82-1.33$ & 0.73 & 0.96 & $0.73-1.26$ & 0.77 \\
\hline Increasing peritumoral eosinophil count & 0.75 & $0.58-0.98$ & 0.04 & 0.70 & $0.52-0.93$ & 0.01 \\
\hline
\end{tabular}

Abbreviation: CI, confidence interval.

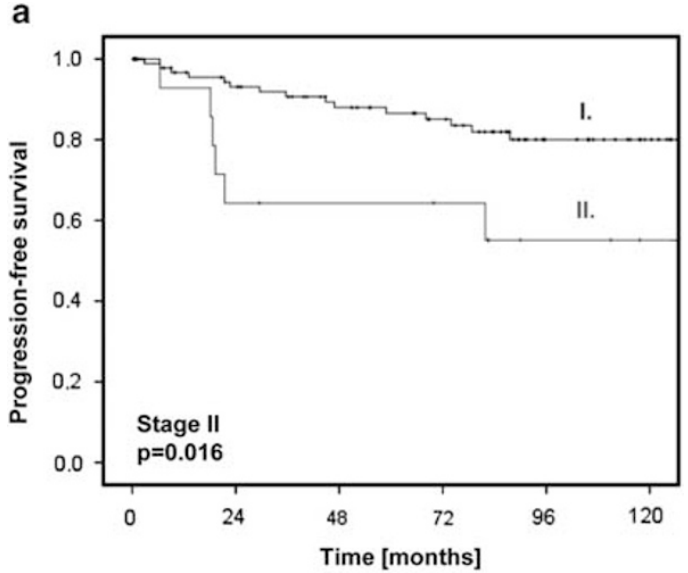

b
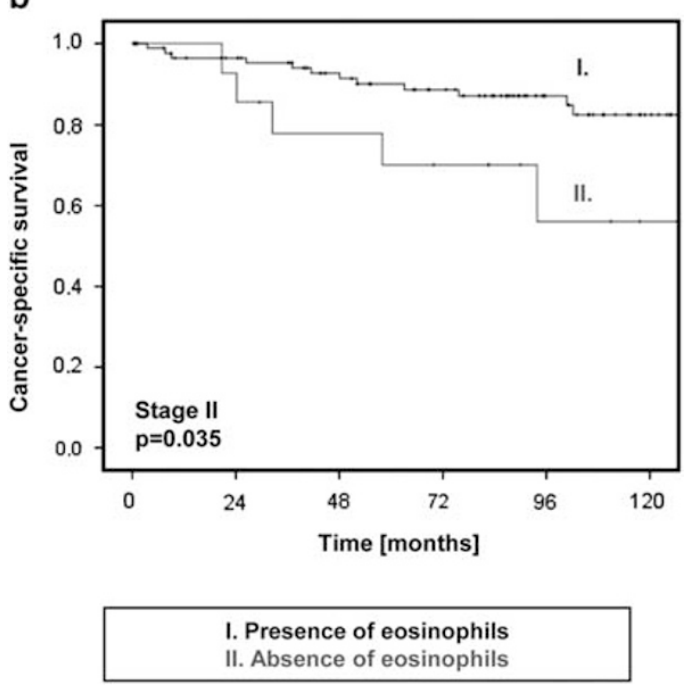

Figure 4 Presence of peritumoral eosinophils is associated with survival in AJCC/UICC stage II colorectal cancers. Kaplan-Meier curves showing progression-free (a) and cancer-specific survival (b) according to presence or absence of peritumoral eosinophils. Comparisons were calculated using log-rank test. $P$-values are two sided. AJCC, American Joint Committee on Cancer; UICC, Union Internationale Contre le Cancer.

\section{Discussion}

According to our data, the presence or an increasing number of eosinophils at the tumor margin is strongly associated with a favorable tumor phenotype as reflected by the TNM classification, tumor cell differentiation, vascular invasion, and tumor budding as well as with improved patients' survival. Most importantly, our results demonstrate that, although the peritumoral eosinophil count correlates with the intensity of the overall inflammatory cell reaction, it is independently associated with outcome.

Given the easy accessibility on hematoxylin and eosin-stained tumor slides, previous studies have addressed the clinicopathological significance of eosinophil infiltration in colorectal cancers. However, comparability between these studies is limited due to differences in scoring systems, location of interest (eg, tumor margin), and adjustment for covariables. Despite these limitations, early studies assessing the number of eosinophils have consistently reported greater numbers in tumors lacking lymph node or distant metastases. ${ }^{17-20}$ Following this, studies have related tumor-infiltrating eosinophils with patient's survival. Fisher et a ${ }^{10}$ reported an association of higher numbers of eosinophils ( $>10$ per 30 oil immersion fields) with better overall survival. However, this effect diminished after adjusting for tumor stage and, surprisingly, no correlation with the overall inflammatory cell reaction was observed in this study. ${ }^{10}$ Nielsen et $a l^{7}$ stratified a cohort of 584 colorectal cancer patients according to the numbers of submucosal eosinophils into four groups and observed an association with improved overall survival of patients independent of tumor stage. Fernandez-Acenero et $a l^{8}$ reported an association with better overall and progression-free survival independent of tumor stage, tumor cell differentiation, and venous invasion in a cohort of 126 colorectal cancer patients. Notably, no association 
Table 3 Cox proportional hazards regression model assessing the prognostic significance of presence of peritumoral eosinophils in conjunction with other well-established tumor parameters regarding progression-free and cancer-specific survival in patients with AJCC/ UICC stage II colorectal cancers $(n=108)$

\begin{tabular}{|c|c|c|c|c|c|c|}
\hline & \multicolumn{3}{|c|}{ Progression-free survival } & \multicolumn{3}{|c|}{ Cancer-specific survival } \\
\hline & Hazard ratio & $95 \% C I$ & $\mathrm{P}$-value & Hazard ratio & $95 \% C I$ & P-value \\
\hline Age $>70$ years & 0.85 & $0.30-2.45$ & 0.63 & 1.09 & $0.31-3.76$ & 0.89 \\
\hline Female gender & 0.34 & $0.10-1.09$ & 0.07 & 0.39 & $0.10-1.46$ & 0.16 \\
\hline Deep tumor invasion (pT4) & 3.10 & $0.70-13.63$ & 0.14 & 5.08 & $1.10-23.41$ & 0.04 \\
\hline Poor tumor differentiation (G3) & 1.35 & $0.50-3.70$ & 0.56 & 1.64 & $0.53-5.06$ & 0.39 \\
\hline Presence of lymphatic invasion & 0.24 & $0.05-1.27$ & 0.09 & 0.48 & $0.09-2.60$ & 0.40 \\
\hline Presence of venous invasion & 2.97 & $0.73-11.00$ & 0.13 & 3.69 & $0.81-16.71$ & 0.09 \\
\hline High tumor budding & 1.75 & $1.10-2.80$ & 0.02 & 1.71 & $1.02-2.84$ & 0.04 \\
\hline Increasing intensity of overall inflammatory cell reaction & 1.14 & $0.60-2.14$ & 0.70 & 1.15 & $0.55-2.41$ & 0.72 \\
\hline Presence of peritumoral eosinophils & 0.24 & $0.07-0.87$ & 0.03 & 0.25 & $0.06-1.02$ & 0.05 \\
\hline
\end{tabular}

Abbreviations: AJCC, American Joint Committee on Cancer; CI, confidence interval; UICC, Union Internationale Contre le Cancer.

with tumor characteristics such as TNM classification or vascular invasion occurred in this study. ${ }^{8}$ However, Fernandez-Acenero et $a l^{8}$ did not consider the localization of eosinophils nor the overall inflammatory cell reaction, whereas Nagtegaal et $a l^{9}$ compared eosinophil infiltration within the tumor center and at the tumor margin in a cohort of 160 colorectal cancer patients. These authors applied a semi-quantitative three-tiered scoring system (combining those tumors lacking eosinophils and with low eosinophil count) and found a less strong correlation between intra- and peritumoral eosinophil count compared with our study $(R=0.26 \quad$ vs $R=0.69) .{ }^{9}$ Although an increasing peritumoral, but not intratumoral, eosinophil count was associated with better overall survival and lower rates of recurrence, this effect was not independent of TNM classification and, importantly, overall inflammatory cell reaction in this study. ${ }^{9}$

Nagtegaal et $a l^{9}$ assessed the inflammatory reaction according to the system proposed by Jass et al, ${ }^{21}$ wherein an inflammatory infiltration at the invasive margin was graded depending on the appearance as a connective tissue lamina following the advancing edge of the tumor. Mainly, the occurrence of lymphocytes was considered when assessing this lamina. ${ }^{21}$ In contrast, the grading scheme according to Klintrup et $a l,{ }^{6}$ which was applied in the present study, includes all types of inflammatory cells in a four-tiered system. The connective tissue lamina as described by Jass et $a l^{21}$ may in fact be reflected by the band-like infiltrate of inflammatory cells at the invasive margin defining score 2 in the KlintrupMäkinen scheme. ${ }^{6}$ The latter scheme has been demonstrated to be associated with survival of colorectal cancer patients in several studies. ${ }^{6,11,22,23}$ Herein, Roxburgh et $a l^{22}$ have compared both scoring systems and found an independent association with patients' survival in node-negative colorectal cancers only for the Klintrup-Mäkinen scheme. Furthermore, the inter-observer concordance was superior for the Klintrup-Mäkinen scheme. ${ }^{23}$

The prognostic importance of the colorectal cancer's microenvironment has recently been reflected by an international effort to develop a host-dependent score ('immunoscore'). This score bases on the assessment of tumor-associated CD3 ${ }^{+}$ and/or $\mathrm{CD}^{+}{ }^{+} \mathrm{T}$ lymphocytes. It was shown to be associated with disease recurrence independent of tumor's stage according to AJCC/UICC. ${ }^{3}$ Furthermore, high numbers of tumor-infiltrating $\mathrm{T}$ lymphocytes with a regulatory phenotype $\left(\mathrm{FOXP}^{+}\right)$were associated with survival in colorectal cancers. ${ }^{24}$ However, it has to be clarified whether lymphocyte subtyping adds additional prognostic information beyond the evaluation of inflammatory cells on routine hematoxylin and eosin-stained slides. ${ }^{5}$

Eosinophils are multifunctional granulocytes and potent effector cells that release several cytotoxic mediators on activation. ${ }^{25}$ Only little evidence exists with respect to the mechanisms of antitumoral activity exhibited by eosinophils. It is unclear whether the antitumoral activity is dependent of a Th2 immune response. Cormier et $a l^{26}$ demonstrated that antitumoral eosinophil activity occurs despite depletion of $\mathrm{CD}^{+}{ }^{+} \mathrm{T}$ lymphocytes in a xenograft animal model. This would suggest that recruitment and activation of eosinophils are driven immediately by the tumor cells. On the contrary, Mattes et $a l^{27}$ showed a strong antitumoral activity of eosinophils mediated by $\mathrm{CD} 4{ }^{+} \mathrm{T}$ lymphocytes following transfection of tumor cells with ovalbumin. An enhanced accumulation of tumorassociated eosinophils has also been observed when tumor cells were transfected with interleukin (IL)-5, whereas eosinophil-deficient IL-5/CCL11 (-/ -) mice have been demonstrated to enable augmented tumorigenicity in xenograft models. ${ }^{28}$ Tumoricidal activation and degranulation of eosinophils seem to depend on stable contacts with colorectal cancer 
a

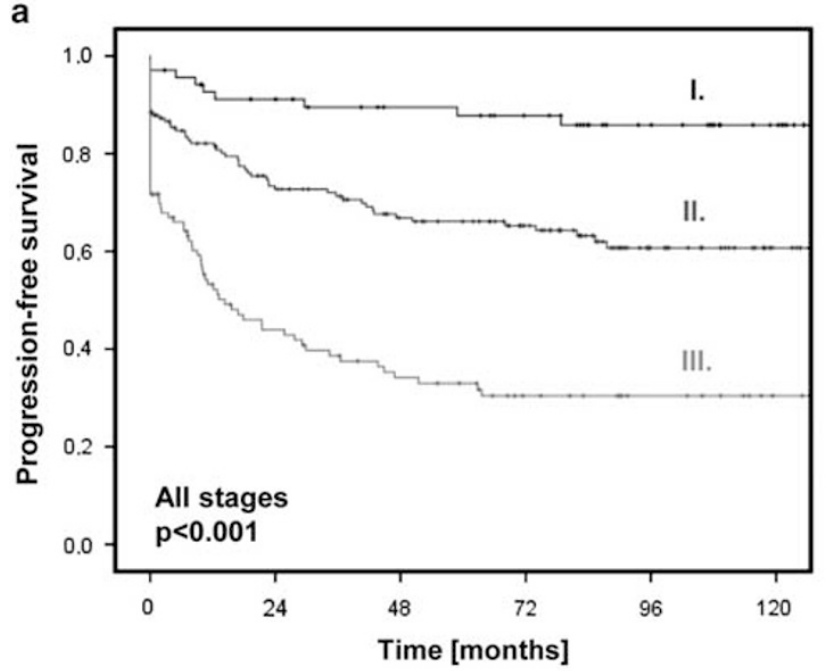

C

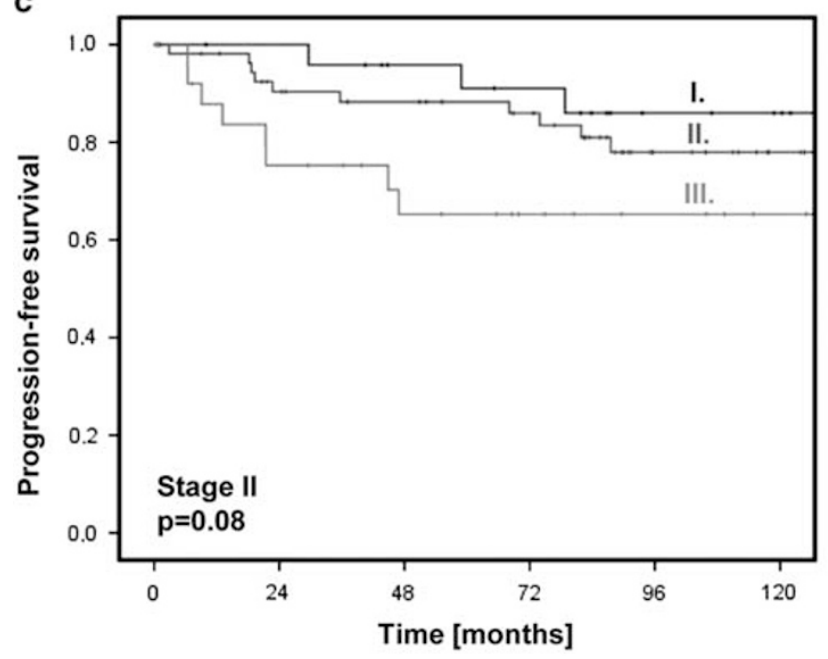

b

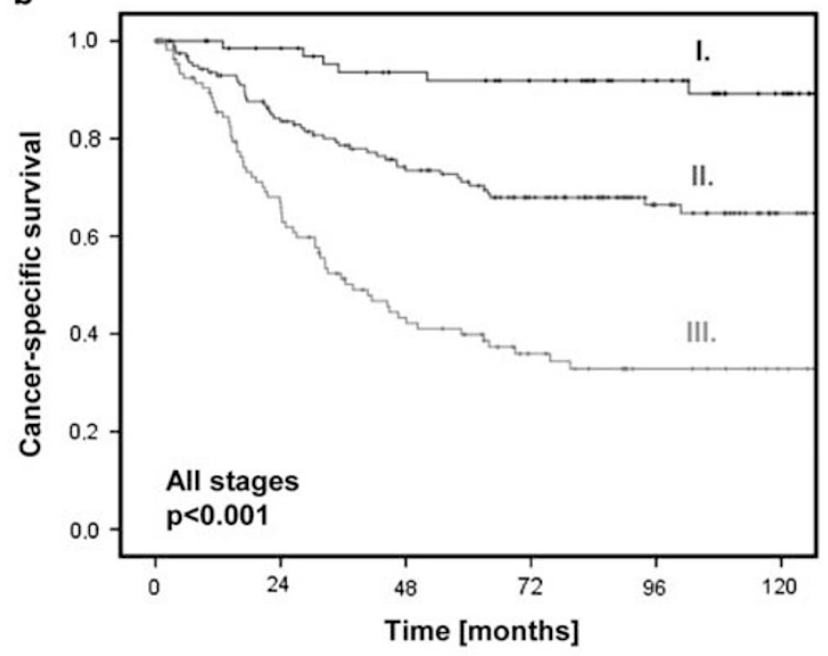

d

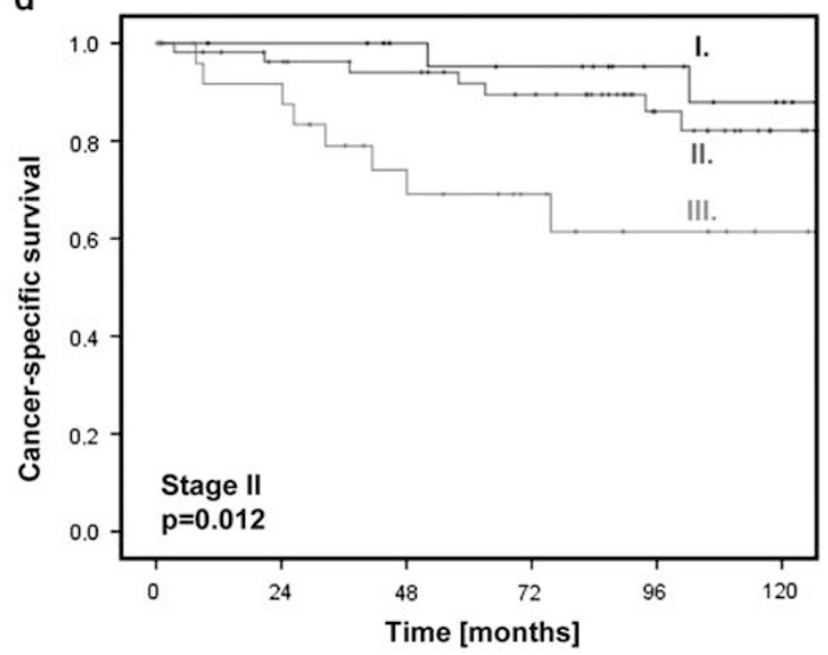

I. Intermediate to high peritumoral eosinophil count and low extent tumor budding

II. Intermediate to high peritumoral eosinophil count and high extent tumor budding as well as absent to low peritumoral eosinophil count and low extent tumor budding

III. Absent to low peritumoral eosinophil count and high extent tumor budding

Figure 5 Combined analyses of peritumoral eosinophil count and extent of tumor cell budding is associated with survival—overall and in AJCC/UICC stage II colorectal cancer. Kaplan-Meier curves showing progression-free (a) and cancer-specific survival (b) according to a combined parameter of peritumoral eosinophil count and extent of tumor cell budding. In patients with AJCC/UICC stage II disease, Kaplan-Meier curves showing progression-free (c) and cancer-specific survival (d) according to a combined parameter of peritumoral eosinophil count and extent of tumor cell budding. Comparisons were calculated using log-rank test. P-values are two sided. AJCC, American Joint Committee on Cancer; UICC, Union Internationale Contre le Cancer.

cells, and secreted cytotoxic proteins may include eosinophil cationic proteins, tumor necrosis factor$\alpha$, and granzyme A. ${ }^{29}$

Reports from immunotherapeutic approaches in patients with epithelial tumors suggest that the accumulation and activity of eosinophils might represent an important mechanism of action. Efficacy of treatment with recombinant $\mathrm{IL}-2$ in advanced cancers was associated with greater numbers of peritumoral eosinophils. ${ }^{30}$ Administration of IL-4 following treatment with IL-2, furthermore, induced eosinophil degranulation in a dose-dependent manner, which was evident by elevated eosinophil's major basic proteins in blood samples along with moderate blood eosinophilia. ${ }^{31,32}$ An eosinophil-dependent antitumoral mechanism of 
IL-4 was supported by animal studies with specific blockage of granulocytes and/or lymphocytes. ${ }^{33}$ More recently, it has been demonstrated that IL-25 (synonym IL-17E), which is also released by eosinophils, exhibits antitumoral activity and leads to eosinphilia in a colorectal cancer xenograft model. ${ }^{34}$ IL-25 may induce the expansion of eosinophils through IL-5 as well as elevated levels of IL-4 and IL-13, but also directly induce apoptosis of cancer cells. ${ }^{35}$

Our study has strengths and limitations. The investigated cohort of colorectal cancer patients is large, with a comparably long follow-up time. Moreover, this cohort represents a random sample of more than 7500 colorectal cancer patients. Review pathology was performed independently by two experienced gastrointestinal pathologists. Foremost are the limitations inherent to retrospective analyses. By applying previously established cutoff values to group eosinophils, we were not able to address the question which is the optimal cutoff in predicting patients' survival. By excluding patients with neoadjuvant chemotherapy, patients with synchronous or metachronous secondary colorectal cancer as well as patients with competitive invasive cancers originating from other sites if metastatic deposits were not assessed by histology, we tried to control the homogeneity of the study population. Nevertheless, the patients included in this study underwent surgical therapy by multiple surgeons from both academic and community settings. Our results still strongly suggest that the peritumoral eosinophil count is a relevant prognostic parameter in patients with colorectal cancer independent of AJCC/UICC stage, which we, in particular, demonstrate for patients with stage II colorectal cancers.

In conclusion, despite the potential to improve risk stratification, a reliable measure of the local inflammatory response is yet to be incorporated into clinical practice. In this regard, evaluation of peritumoral eosinophils is a promising tool, particularly as it is readily assessable already on hematoxylin and eosin-stained tumor slides. The number of peritumoral eosinophils seems to have a strong favorable impact on the prognosis of patients with colorectal cancer, which is independent of tumor stage and the intensity of the overall inflammatory cell reaction.

\section{Disclosure/conflict of interest}

The authors declare no conflict of Interest.

\section{References}

1 Brenner H, Kloor M, Pox CP. Colorectal cancer. Lancet 2013;383:1490-1502.

2 Compton CC. Optimal pathologic staging: defining stage II disease. Clin Cancer Res 2007;13:6862s-6870ss.
3 Galon J, Mlecnik B, Bindea G, et al. Towards the introduction of the 'Immunoscore' in the classification of malignant tumours. J Pathol 2014;232:199-209.

4 Hanahan D, Weinberg RA. Hallmarks of cancer: the next generation. Cell 2011;144:646-674.

5 Roxburgh CS, McMillan DC. The role of the in situ local inflammatory response in predicting recurrence and survival in patients with primary operable colorectal cancer. Cancer Treat Rev 2012;38: 451-466.

6 Klintrup K, Makinen JM, Kauppila S, et al. Inflammation and prognosis in colorectal cancer. Eur J Cancer 2005;41:2645-2654.

7 Nielsen HJ, Hansen U, Christensen IJ, et al. Independent prognostic value of eosinophil and mast cell infiltration in colorectal cancer tissue. J Pathol 1999;189:487-495.

8 Fernandez-Acenero MJ, Galindo-Gallego M, Sanz J, et al. Prognostic influence of tumor-associated eosinophilic infiltrate in colorectal carcinoma. Cancer 2000;88:1544-1548.

9 Nagtegaal ID, Marijnen CA, Kranenbarg EK, et al. Local and distant recurrences in rectal cancer patients are predicted by the nonspecific immune response; specific immune response has only a systemic effect-a histopathological and immunohistochemical study. BMC Cancer 2001;1:7.

10 Fisher ER, Paik SM, Rockette H, et al. Prognostic significance of eosinophils and mast cells in rectal cancer: findings from the National Surgical Adjuvant Breast and Bowel Project (protocol R-01). Hum Pathol 1989;20:159-163.

11 Richards CH, Flegg KM, Roxburgh CS, et al. The relationships between cellular components of the peritumoural inflammatory response, clinicopathological characteristics and survival in patients with primary operable colorectal cancer. $\mathrm{Br} \mathrm{J}$ Cancer 2012;106:2010-2015.

12 Moertel CG, Fleming TR, Macdonald JS, et al. Levamisole and fluorouracil for adjuvant therapy of resected colon carcinoma. N Engl J Med 1990;322:352-358.

13 Sobin L, Gospodarowicz MK, Wittekind C (eds) TNM Classification of Malignant Tumors, 7th edn. WileyBlackwell: West Sussex, UK, 2010.

14 Hamilton SR, Bosman FT, Boffetta P. Carcinoma of the colon and rectum. In: Bosman FT, Carneiro F, Hruban $\mathrm{RH}$, et al. (eds) World Health Organization Classification of Tumours of the Digestive System. IARC: Lyon, France; 2010, pp 131-181.

15 Ueno H, Murphy J, Jass JR, et al. Tumour 'budding' as an index to estimate the potential of aggressiveness in rectal cancer. Histopathology 2002;40:127-132.

16 Hanahan D, Coussens LM. Accessories to the crime: functions of cells recruited to the tumor microenvironment. Cancer Cell 2012;21:309-322.

17 McGinnis MC, Bradley EL Jr, Pretlow TP, et al. Correlation of stromal cells by morphometric analysis with metastatic behavior of human colonic carcinoma. Cancer Res 1989;49:5989-5993.

18 Pretlow TP, Boohaker EA, Pitts AM, et al. Heterogeneity and subcompartmentalization in the distribution of eosinophils in human colonic carcinomas. Am J Pathol 1984;116:207-213.

19 Pretlow TP, Keith EF, Cryar AK, et al. Eosinophil infiltration of human colonic carcinomas as a prognostic indicator. Cancer Res 1983;43:2997-3000.

20 Luebbers EL, Pretlow TP, Emancipator SN, et al. Heterogeneity and prognostic significance of macrophages 
in human colonic carcinomas. Cancer Res 1985;45: 5196-5200.

21 Jass JR. Lymphocytic infiltration and survival in rectal cancer. J Clin Pathol 1986;39:585-589.

22 Roxburgh CS, Salmond JM, Horgan PG, et al. Tumour inflammatory infiltrate predicts survival following curative resection for node-negative colorectal cancer. Eur J Cancer 2009;45:2138-2145.

23 Roxburgh CS, Salmond JM, Horgan PG, et al. Comparison of the prognostic value of inflammation-based pathologic and biochemical criteria in patients undergoing potentially curative resection for colorectal cancer. Ann Surg 2009;249:788-793.

24 Salama P, Phillips M, Grieu F, et al. Tumor-infiltrating FOXP3 + T regulatory cells show strong prognostic significance in colorectal cancer. J Clin Oncol 2009;27:186-192.

25 Jacobsen EA, Taranova AG, Lee NA, et al. Eosinophils: singularly destructive effector cells or purveyors of immunoregulation? J Allergy Clin Immunol 2007;119:1313-1320.

26 Cormier SA, Taranova AG, Bedient C, et al. Pivotal Advance: eosinophil infiltration of solid tumors is an early and persistent inflammatory host response. J Leukoc Biol 2006;79:1131-1139.

27 Mattes J, Hulett M, Xie W, et al. Immunotherapy of cytotoxic T cell-resistant tumors by T helper 2 cells: an eotaxin and STAT6-dependent process. J Exp Med 2003;197:387-393.

28 Simson L, Ellyard JI, Dent LA, et al. Regulation of carcinogenesis by IL-5 and CCL11: a potential role for eosinophils in tumor immune surveillance. J Immunol 2007;178:4222-4229.

29 Legrand F, Driss V, Delbeke M, et al. Human eosinophils exert TNF-alpha and granzyme A-mediated tumoricidal activity toward colon carcinoma cells. J Immunol 2010;185:7443-7451.

30 Arinaga S, Karimine N, Takamuku K, et al. Correlation of eosinophilia with clinical response in patients with advanced carcinoma treated with low-dose recombinant interleukin-2 and mitomycin C. Cancer Immunol Immunother 1992;35:246-250.

31 Sosman JA, Bartemes K, Offord KP, et al. Evidence for eosinophil activation in cancer patients receiving recombinant interleukin-4: effects of interleukin-4 alone and following interleukin-2 administration. Clin Cancer Res 1995;1:805-812.

32 Sosman JA, Fisher SG, Kefer C, et al. A phase I trial of continuous infusion interleukin-4 (IL-4) alone and following interleukin-2 (IL-2) in cancer patients. Ann Oncol 1994;5:447-452.

33 Tepper RI, Coffman RL, Leder P. An eosinophildependent mechanism for the antitumor effect of interleukin-4. Science 1992;257:548-551.

34 Benatar T, Cao MY, Lee Y, et al. IL-17E, a proinflammatory cytokine, has antitumor efficacy against several tumor types in vivo. Cancer Immunol Immunother 2010;59:805-817.

35 Furuta S, Jeng YM, Zhou L, et al. IL-25 causes apoptosis of IL-25R-expressing breast cancer cells without toxicity to nonmalignant cells. Sci Transl Med 2011;3:78ra31. 